The Electronic Gharge $e$.

Prof. A. S. Eddington has recently (Proc. Roy. Soc., A, 122, 358; Jan. 1929) deduced a theoretical value of 136 for the well-known ratio $h c / 2 \pi e^{2}$. The reciprocal of this ratio is usually denoted 'the finestructure constant a.' Without presuming in any way to judge the theory on which this value is derived, I should like to make a few remarks as to the numerical result. The value of the velocity of light $c$ is known with great accuracy $(c=2.99796 \pm 0.00004)$ On the other hand, the value of the Planck constant $h$ depends primarily upon the value of the electronic charge $e$, and the probable error in $h$ is almost entirely due to the probable error in $e$. Every method for evaluating $h$ involves $e$ to a positive power varying from unity to two. The average power depends upon the adopted relative weighting of the different methods. These facts regarding the connexion of $e$ and $h I$ discussed some years ago (Phys. Rev., 14, 361; 1919).

I am at the present moment just finishing a critical investigation of the probable values of the general constants of physical science, and a detailed account of this work will be published shortly. At the present time my adopted value of $h$ depends, in the mean, on the 1.236 power of $e$. Hence the ratio $h / e^{2}$ varies as $1 / e^{0.764}$. The change in this ratio demanded by Eddington's theory is approximately 1 per cent downward $(0.94$ per cent, using my own adopted values of $e, h$, and $c)$. Accordingly, such a change requires an increase in $e$ of approximately one and one-quarter per cent, and a resulting increase in $h$ of about one and one-half per cent, in contrast to a one-half per cent increase in $e$ (and no change in $h$ ) assumed by Eddington as required. In my opinion the commonly accepted value of $e$ has a probable error of roughly 0.1 per cent, and it is accordingly extremely improbable that the true error is more than twelve times as great.

University of California, Feb. 2.

RAYMOND T. BIRGE.

\section{The Boundary of the Solar Chromosphere.}

The question of the sudden ending of the chromosphere or its gradual fading away in accordance with Prof. Milne's theoretical views may not yet be settled finally by observation. Mr. R. W. Gurney is, however, under a misapprehension (Nature, Feb. 16, p. 240) in thinking that the bright $K$ line studied by Mr. P. A. Taylor and Mr. McCrea up to a height of nearly $100,000 \mathrm{~km}$. above the sun's limb was thought to be an ordinary chromospheric line. The tangential slit happened to fall across a high prominence and the measures refer to the portions of the slit lying on the prominence, which gave a regular fading away with height; one or two obvious brightenings had to be ignored, where structure in the prominence complicated the issue. These points were easily recognised in the picture of the prominence shown in the second flash spectrum which was obtained with an objective prism.

The difficulty of the scattering of light in our atmosphere is not easy to meet, but evidence from our other plates, for example, the objective prism spectra, does not point to any serious trouble in our case. We had the good fortune to observe the sun in a perfectly clear hole in a somewhat cloudy sky. Messrs. Miller and Marriott, half a mile away, observed through thin haze. The heights of the chromospheric lines proper, which we published, were taken from the arcs given by the objective prism spectrograms of the flash, and these would not be seriously affected by light scattering. Incidentally, it may be added, they are not inconsistent with Mr. Gurney's views.

Feb. 18. F. J. M. Stratton. C. R. Davidson.

\section{An Isotope of Oxygen, Mass 18.}

The weak doublets of the atmospheric absorption bands of oxygen have been found to originate from a molecule consisting of an oxygen atom of mass 18 combined with one of mass 16. The interpretation recently published by Mulliken (Phys. Rev., 32, 880 ; 1928) for the strong bands holds in every detail for the weak band. The isotopic rotation-zero point vibration doublets have been calculated by means of the equations of Loomis (Bull. Nat. Res. Council, 2, chap. v.; 1926) and the atmospheric absorption data of Dieke and Babcock (Proc. N.A.S., 13, 670 ; 1927). The vibrational frequency used for the lower state is that calculated by Birge (Bull. Nat. Res. Council, 2, $232 ; 1927)$ from the available data. From the above, the formulæ for the separation of the isotopic doublets in the four $P$ and four $R$ branches is as follows:

$$
\begin{aligned}
\Delta \nu_{P} & =2 \cdot 12+0.0556\left[B^{\prime \prime} m^{2}-\beta^{\prime \prime} m^{4}\right. \\
\Delta \nu_{R} & =2 \cdot 12+0.0556\left[B^{\prime \prime} m^{2}-\beta^{\prime \prime}(m-1)^{4}+\beta^{\prime}(m-1)^{4}\right] \\
m & =\frac{3}{2}, \frac{7}{2}, \frac{11}{2}, \text { etc. }
\end{aligned}
$$

The constants as given by Dieke and Babcock are

$$
\begin{array}{ll}
B^{\prime \prime}=1.438 & \beta^{\prime \prime}=6.31 \times 10^{-6} \\
B^{\prime}=1.390 & \beta^{\prime}=5 \cdot 75 \times 10^{-6}
\end{array}
$$

The average deviation of observed minus calculated separations is $-0.05 \mathrm{~cm} .^{-1}$. The maximum deviation is $-0.13 \mathrm{~cm}^{-1}$. This is well within the limit of accuracy of the data. No other isotope of oxygen combined with an atom of mass 16 will satisfy the data. The data show that the normal state of the oxygen molecule has one-half unit of vibration in agreement with the wave mechanics theory.

W. F. Giauque.

H. L. JoHNSTON.

Department of Chemistry, University of California, Berkeley, California.

Intercombinations in the Arc Spectrum of Carbon.

Prof. A. Fowler and E. W. H. Selwyn have recently classified the lines of the arc spectrum of carbon, and identified triplet as well as singlet terms, but no intercombinations have apparently been

\begin{tabular}{|c|c|c|}
\hline 51313 & $\begin{array}{l}\text { Classification. } \\
{ }^{1} \bar{D}_{2}-{ }^{3} \bar{P}_{1}\end{array}$ & $\begin{array}{c}\text { Transition. } \\
\left(2 L_{2} \leftarrow-L_{2} M_{1}\right)\end{array}$ \\
\hline 51356 & ${ }^{1} \bar{D}_{2}-{ }^{3} \bar{P}_{2}$ & $(\quad, \quad)$ \\
\hline 39862 & ${ }^{1} \overline{S_{0}}-{ }^{3} \bar{P}_{1}$ & , \\
\hline
\end{tabular}
obtained. Recently I took a heavy arc spectrum of Acheson graphite in the region $\lambda 2000$, and obtained a number of lines, some new, and others recorded by previous observers like McLennan, Hutchinson, and others. I was able to identify the following intercombination lines:

This enables us to calculate the exact differences between the fundamental levels ${ }^{3} P_{012}$ and ${ }^{1} D_{2},{ }^{1} S_{0}$ of $2 L_{2}$. We get ${ }^{3} \bar{P}_{1}-1 \bar{S}_{0}=20474$, while according to Fowler and Selwyn it is 21142. Taking Fowler's ${ }^{1} D_{2}$ value as the more correct, the values of fundamental ${ }^{3} P$ terms have to be decreased by $667 \mathrm{~cm}^{-1}$. We have also lines conforming to the inner-transitions $\left(L_{2} L_{2} \leftarrow L_{1} 3 L_{2}\right)$; from the new lines I have also obtained identification of some of the $\left(2 L_{1} L_{2} M_{1}\right.$ $\leftarrow-L_{1} 2 L_{2} M_{1}$ ) transition lines.

The frequency difference ${ }^{3} P_{1}-1 S_{0}=20474$ corresponds to the wave-length $\lambda 4884 \cdot 2$, and I could get no such line in the coronal spectrum.

Physics Department, Dattatraya Shridhar Jog. Allahabad University.

No. 3096, VoL.' 123] 